\title{
Numerical solution of three-dimensional rectangular submerged jets with the evidence of the undisturbed region of flow
}

\section{Angelino, A. Boghi \& F. Gori}

To cite this article: M. Angelino, A. Boghi \& F. Gori (2016): Numerical solution of threedimensional rectangular submerged jets with the evidence of the undisturbed region of flow, Numerical Heat Transfer, Part A: Applications, DOI: 10.1080/10407782.2016.1214494

To link to this article: http://dx.doi.org/10.1080/10407782.2016.1214494

曲 Published online: 20 Sep 2016.

Submit your article to this journal $\pi$

Џll Article views: 15

Q View related articles $\asymp$

View Crossmark data $\nearrow$ 


\title{
Numerical solution of three-dimensional rectangular submerged jets with the evidence of the undisturbed region of flow
}

\author{
M. Angelino ${ }^{a}$, A. Boghi ${ }^{b}$, and F. Gori ${ }^{\mathrm{c}}$ \\ aLoughborough University, Aeronautical and Automotive Engineering, Loughborough, UK; ${ }^{b}$ Cranfield University, \\ School of Engineering, Environment and Agrifood, Cranfield, UK; 'Department of Industrial Engineering, University of \\ Rome "Tor Vergata", Rome, Italy
}

\begin{abstract}
The evolution of turbulent rectangular submerged free jets has been investigated numerically with a two-dimensional (2D) approach by the present authors and, by using the large eddy simulations (LES) at several Reynolds numbers. The average numerical results confirmed the presence of the undisturbed region of flow (URF) located between the slot exit and the beginning of the potential core region (PCR) previously observed experimentally at the University of Rome "Tor Vergata" by Gori and coworkers. The 2D study of the present authors carried out under the conditions previously investigated in the literature, showed that the URF has a self-similar behavior, and proposed a new law for the evolution of the momentum. The present paper extends the LES to three-dimensional (3D) rectangular submerged free jets, in the range from $R e=5,000$ to $\operatorname{Re}=40,000$, showing that the self-similar behavior of URF is also present in the $3 D$ numerical simulations, as well as in the PCR and in the fully developed region (FDR).
\end{abstract}

\section{ARTICLE HISTORY}

Received 30 March 2016

Accepted 23 June 2016

\section{Introduction}

The interpretation of the evolution of turbulent rectangular submerged free jets, investigated in the last decades, can be summarized by the interaction of the jet with the stagnant fluid just after the slot exit [2], defining two regions of flow: the potential core region (PCR) and the fully developed region (FDR). The flow in the PCR and FDR was studied theoretically by Tollmien [3] and Görtler [4], who found self-similar velocity profiles in the two regions of flow. The equations of $[2,3]$ describing their evolutions have been confirmed experimentally for the PCR [5-9] and the FDR [10-13].

The experiments carried out at the University of Rome "Tor Vergata" by Gori and coworkers investigated the flow evolution of a rectangular jet of air, with hot wire anemometer and shadowgraph visualizations $[14,15]$, hot film anemometer and shadowgraph visualizations, [16-18], and particle image velocimetry (PIV) $[19,20]$. The presence of a third region of flow, which has been called undisturbed region of flow (URF), has been observed in the average visualizations and measurements [19], and located between the slot exit and the beginning of the PCR. The average experiments established that its length diminishes with the increasing Reynolds number. Further experiments with the PIV on the instantaneous flow evolution [20] have shown that the URF can be further divided into two new types of flow: the negligible disturbances flow (NDF) and the small disturbances flow (SDF). In the NDF the jet height remains almost constant, as well as the velocity and the turbulence profiles, while in the SDF, the jet height evidences small contractions or expansions, but without the formation of vortices.

CONTACT F. Gori gori@uniroma2.it $\Theta$ Department of Industrial Engineering, University of Rome "Tor Vergata", Via del Politecnico 1, 00133 Rome, Italy.

Color versions of one or more of the figures in the article can be found online at www.tandfonline.com/unht. 


\section{Nomenclature}

$\begin{array}{ll}\text { Latin } & \\ a & \text { generic field or similarity coefficient } \\ c & \text { Tollmien coefficient } \\ D & \text { diameter } \\ e & \text { Görtler coefficient } \\ f & \text { similarity function } \\ g & \text { spatial filter function } \\ h & \text { dimensionless half-height of the slot } \\ \mathrm{H} & \text { dimensional height of the slot } \\ k & \text { kinetic energy } \\ p & \text { instantaneous static pressure } \\ P & \text { mean static pressure } \\ \mathrm{Re} & \text { Reynolds number } \\ S & \text { shear rate } \\ t & \text { time } \\ u & \text { instantaneous axial velocity } \\ U & \text { mean axial velocity } \\ v & \text { instantaneous cross-stream velocity } \\ V & \text { mean cross-stream velocity } \\ v & \text { instantaneous cross-stream velocity }\end{array}$

$\begin{array}{ll}x & \text { streamwise coordinate } \\ y & \text { cross-stream coordinate } \\ \text { Greek } & \\ \delta & \text { identity tensor } \\ \Delta & \text { filter width } \\ \zeta & \text { Tollmien similarity variable } \\ \eta & \text { similarity variable } \\ \nu & \text { kinematic viscosity } \\ \xi & \text { Görtler similarity variable } \\ \tau & \text { sub-grid stress tensor } \\ \psi & \text { stream-function } \\ \Psi & \text { stream-function of Tollmien and Görtler } \\ \Omega & \text { domain dimension } \\ \text { Subscripts } \\ 0 & \text { initial } \\ h & \text { hydraulic } \\ \text { in } & \text { inlet } \\ \text { sgs } & \text { sub-grid scale } \\ T & \text { turbulent }\end{array}$

Looking back to previous experiments of the literature it is possible to recognize that a region of flow, which can be considered a URF, was present in the experiments of [21-23] for certain inlet conditions. In other experimental works [24-27], direct numerical simulations (DNS) [28, 29] and large eddy simulations (LES) [30], the URF was evidenced but not discussed.

The URF has not been investigated numerically so far, except in [31], where preliminary numerical solutions, obtained with the RANS modeling, are compared with the experimental shadowgraph visualizations at $\operatorname{Re}=25,000$ and 60,000. The study preceding the present work, from the same present authors [1], has carried out a series of LES at several Reynolds numbers in the range from $\operatorname{Re}=5,000$ to $\operatorname{Re}=40,000$, to verify that the URF occurs in the conditions previously given by the literature [2] in a two-dimensional (2D) case.

The numerical analysis can give further information, besides the experiments, which can contribute to a better understanding of the phenomenon of heat transfer upon a cylinder due to jet flow [32-35]. This problem depends on several variables, such as the presence of multiple cylinders in a row $[36,37]$, fins [38-42], the level of turbulence in the jet $[43,44]$, the slot to cylinder diameter ratios [45, 46], and special conditions like pulsating jets [47].

This work presents the results of three-dimensional (3D) LES of a rectangular free jet, at several Reynolds numbers in the range from $\operatorname{Re}=5,000$ to $\operatorname{Re}=40,000$, which extends the $2 \mathrm{D}$ approach [1], in order to show that even in the 3D case it is possible to define self-similar relations for the URF, and to assess that the theoretical model is valid even in the presence of a turbulent profile on the slot exit.

\section{Numerical method}

\subsection{Governing equations}

In the LES approach, the governing equations are derived by filtering the Navier-Stokes equations. This is particularly easy in case of an incompressible fluid. The filtered variables are indicated by the superscript $\sim$. Applying the filtering operation to the mass and momentum equations, the following equations are obtained:

$$
\frac{\partial \tilde{u}_{i}}{\partial x_{i}}=0
$$




$$
\frac{\partial \tilde{u}_{i}}{\partial t}+\frac{\partial}{\partial x_{j}}\left(\tilde{u}_{i} \tilde{u}_{j}+\tilde{p} \delta_{i j}-\frac{2}{\operatorname{Re}} \tilde{S}_{i j}\right)=\frac{\partial \tau_{i j}^{s g s}}{\partial x_{j}}
$$

where $u_{i}$ is the velocity vector, $p$ the static pressure, and $\delta_{i j}$ the identity tensor. The Reynolds number Re is defined as

$$
\operatorname{Re}=\frac{U_{\text {in }} D_{h}}{\nu}
$$

where $U_{i n}$ is the inlet velocity, $D_{h}$ the hydraulic diameter, defined as four times the ratio between the slot area and its perimeter, and $v$ the fluid kinematic viscosity. The shear rate tensor, $S_{i j}$, is defined as

$$
\tilde{S}_{i j}=\frac{1}{2}\left(\frac{\partial \tilde{u}_{i}}{\partial x_{j}}+\frac{\partial \tilde{u}_{j}}{\partial x_{i}}\right)-\frac{1}{3} \frac{\partial \tilde{u}_{k}}{\partial x_{k}} \delta_{i j}
$$

The sub-grid stress tensor $\tau_{i j}^{s g s}$ is

$$
\tau_{i j}^{s g s}=2 \nu_{s g s} \tilde{S}_{i j}-\frac{2}{3} k_{s g s} \delta_{i j}
$$

The sub-grid viscosity is

$$
\nu_{s g s}=C_{S} \Delta^{2} \tilde{S}
$$

The sub-grid kinetic energy is

$$
k_{s g s}=C_{I} \Delta^{2} \tilde{S}^{2}
$$

The filtered shear rate is

$$
\tilde{S}=\sqrt{2 \tilde{S}_{i j} \tilde{S}_{i j}}
$$

The filter width is

$$
\Delta=\sqrt{\Delta_{m} \Delta_{m}}
$$

with $\Delta_{k}$ the box filter width in the $m$ th direction. The dynamic Smagorinsky model is employed in this study.

\subsection{Computational details}

The simulations are realized with the pisoFoam finite-volume solver of the open-source code OpenFOAM, which solves the Navier-Stokes transient incompressible equations via the PISO algorithm. The $3 \mathrm{D}$ grid is generated with blockMesh, the OpenFOAM utility for mesh generation, at all the Reynolds numbers. The geometry is made of a rectangular box, 2-diameter long in the transverse direction $(z), 3$ diameters in the vertical direction $(y)$, and 6.5 diameters in the axial direction $(x)$. The grid is uniform in the $z$ direction, and stretched in the $x$ and $y$ directions near the slot exit, although the minimum and maximum grid stencils are of the same order of magnitude. In the $z$ direction the stencil is $\Delta z=0.0197$ times the hydraulic diameters, while in the $x$ and $y$ directions they are $(\Delta x, \Delta y) \in[0.0074,0.0294]$ times the hydraulic diameters. The grid is made of $442 \times 262 \times 102$ points. The explicit time integration scheme is of the second order backward, and of the second order central for the spatial derivatives. The time step in each simulation satisfies the condition CFL $<0.5$. The filter chosen in OpenFOAM is the "simple" one, which interpolates the field in a given point with its neighboring cells. The filter amplitude is the cubic root of the cell volume.

A wall is present above and below the slot, with a thickness of 0.176 hydraulic diameters, as in the experiments $[19,20]$. The total pressure is imposed on the outlet boundaries, while the velocity 
boundary condition changes according to its direction. If the fluid flows out of the domain a zero gradient condition is imposed for the velocity, otherwise the velocity is obtained from the patchface normal component of the internal-cell value. This boundary condition in OpenFOAM is known as "pressureInletOutletVelocity". A periodic boundary condition is imposed in the span-wise direction.

The simulations are carried out for 30 flow times, being the "flow time" the ratio between the domain length and the axial velocity on the slot exit. The steady state is assumed to be reached after 10 flow times, while the following 20 flow times are employed to average the instantaneous variables and to obtain the mean turbulent properties.

A top-hat velocity profile is assumed on the slot exit, in analogy with the experiments of [2], and with the addition of some disturbances in order to trigger the turbulence in the 3D simulations. A small perturbation of $1 \%$ in the amplitude of the velocity profile is enough to produce a 3D flow. The technique proposed by Lee et al. [48] is employed, with the advantage of injecting the disturbance in the low and intermediate wave number ranges, where it cannot be immediately damped.

Let us decompose the discrete perturbation velocity field $u_{k}$ in Fourier series:

$$
u_{k}=\frac{1}{N} \sum_{n=0}^{N-1} U_{n} e^{2 \pi i k n / N}
$$

where $U_{n}$ is the complex Fourier mode, which can be written as $U_{n}=\left|U_{n}\right| e^{i \varphi_{n}}$. According to the Parseval theorem

$$
\sum_{n=0}^{N-1} E_{n}=\sum_{m=0}^{N-1}\left|u_{m}\right|^{2}=\frac{1}{N} \sum_{n=0}^{N-1}\left|U_{n}\right|^{2}
$$

In order to generate a perturbation, which is able to reproduce the turbulent energy spectrum, it is assumed that $\left|U_{n}\right|=\sqrt{N E_{n}}$ and $\varphi_{n}$ is a random function of $n$. The turbulent spectrum is given by

$$
E(k) \propto k^{4} e^{-2\left(\frac{k}{k_{0}}\right)^{2}}
$$

with

$$
k=\sqrt{k_{x}^{2}+k_{y}^{2}+k_{z}^{2}}
$$

The inlet perturbation field is assumed to act in three directions.

The simulations have been performed on the Blue Gene/Q system "Fermi" of the Italian supercomputing center CINECA, having requested around 200,000 CPU hours for all the simulations.

\section{Results and discussion}

The results are presented for the four Reynolds numbers and all the variables are expressed in non-dimensional form.

\subsection{Instantaneous variables}

The iso-surfaces of the instantaneous vorticity magnitude are reported in Figure 1 at the four Reynolds numbers investigated. Despite the presence of a $1 \%$ perturbation in the amplitude of the velocity profile, the free shear layer shows an initial undisturbed behavior. A plausible explanation is that on the slot exit, i.e., at the interface between jet and stagnant air, the velocity gradient is so steep that the turbulent stresses are negligible compared to the viscous ones. The Kelvin-Helmholtz 

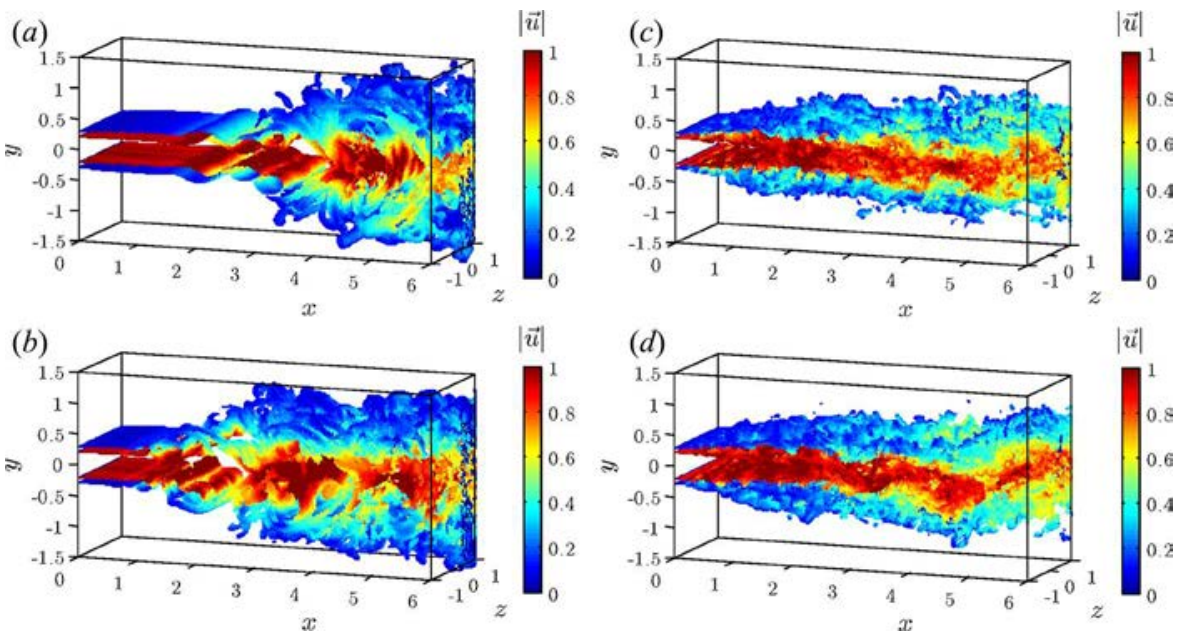

Figure 1. Instantaneous vorticity iso-surfaces colored by instantaneous velocity magnitude: $(a) \operatorname{Re}=5,000 ;(b) \operatorname{Re}=10,000 ;(c) \operatorname{Re}$ $=20,000 ;(d) \operatorname{Re}=40,000$.

instability appears in the free shear layer at different times and positions, according to the Reynolds number. The higher is the Reynolds number, the earlier the roller vortex structures form and the closer they are to the slot exit. This is in agreement with the linear theory of the Kelvin-Helmholtz instability [49], which prescribes that the wave number of the resonant harmonic is proportional to the ratio of the frequency to the mean velocity, so that a low wave number perturbation is enough to trigger the instabilities at high Reynolds number flows. Also, the vortices structure appears to be bigger at smaller Reynolds numbers.

The roller vortex structures develop symmetrically and travel downstream at the speed of the convection velocity. The $z$ component of the vorticity vector has opposite signs above and below the centerline, which means that the vortices above the centerline roll in the anticlockwise direction while the vortices below the centerline roll in the clockwise direction. This cannot be seen in Figure 1 because it shows the iso-surfaces of the vorticity magnitude to clarify the jet shape. At a certain distance downstream the beginning of the Kelvin-Helmholtz instability, the roller vortices eventually pair to form a bigger roller structure and the merging of the shear layers, which triggers the asymmetry in the flow. It is possible to consider the formation of the first Kelvin-Helmholtz instability as the beginning of the PCR, and the beginning of the vortex pairing as its end. In the PCR the resonant sub-harmonic is weak but, as the roller structures travel and pair, it becomes strong enough to activate the asymmetric modes, which trigger the evolution of the coherent structures. In the PCR, three symmetric couples of counter-rotating vortices can be identified, for all the Reynolds numbers.

From time to time, different couples of vortices pair to form larger ones, but this phenomenon stops downstream, where the FDR begins. At the beginning of the FDR the vortices break, by virtue of the vortex-stretching, into very small structures which form a turbulent wake. This is the main difference with the numerical results of the $2 \mathrm{D}$ case [1], where this region is not present, but the large eddies occupy the entire domain, forming an asymmetric pattern. The comparison of the present results with the $2 \mathrm{D}$ case [1] confirms that even in the presence of a perturbation in the amplitude of the velocity profile, there is an NDF immediately downstream the slot exit, where the jet height remains almost constant, followed by the SDF, where the jet height evidences small contractions or expansions but without the formation of vortices, as observed experimentally in [20]. The sum of the lengths of NDF and SDF is about the same of the URF, which has been defined for the average variables. The lengths of NDF and SDF, or URF, decrease with the increase of the Reynolds number, being quite consistent at $\operatorname{Re}=5,000$, a little smaller at 10,000, and quite small at $\operatorname{Re}=20,000$ and 40,000. 
Figure 2 presents the instantaneous isobaric surfaces, shown by different colors, where blue is indicating the low pressure zones, as well as the instantaneous velocity, which is shown by vectors. The jet undergoes a series of pressure fluctuations across its section, i.e., in the transverse direction, and downstream, i.e., in the streamwise direction. The fluid presents a series of accelerations and decelerations in the streamwise direction, especially on the centerline.

The NDF and SDF are clearly visible after the slot exit, as the regions where the velocity vectors are almost unidirectional and constant. The pressure changes on the two borders of the jet produce symmetric variations of the cross section and correspond to symmetric vortices. The jet section decreases in correspondence to the vortices, and increases between the different vortex pairs, due to the conservations of mass, which implies an increase in the velocity where the section reduces. The Bernoulli equation justifies a pressure increase where the velocity decreases.

Figure $2 a$, at $\operatorname{Re}=5,000$, shows that the first three concentric isobars along the shear layer present a slight pressure decrease around $x=1.9$, corresponding to a slight contraction of the cross section, also shown in Figure $1 a$. The two following isobars, around $x=2.4$, on the contrary, present an increase, which can be considered responsible for the expulsion of the fluid from the core of the jet. The formation of the first vortex pair, around $x=3.4$, is generated because the fluid, after the expulsion, is slowed down by the external small velocity of the stagnant fluid. Around $x=5$ the cross section undergoes a strong contraction, after which the end of the potential core is expected.

Figure $2 b$ presents the results for $\mathrm{Re}=10,000$. The pattern presents some similarities with $\mathrm{Re}$ $=5,000$, Figure $2 a$, except for the lengths of the NDF and SDF, which are shorter, and the first vortex pair appears earlier, around $x=1.5$. The vortices appear almost symmetrical along the jet axis with an alternate pattern, and regions of low and high pressure are visible. The number of vortex pairs is higher compared to the previous case, and two big spots of high pressure are clearly visible around $x=3.5$. The velocity vectors at the boundary layer roll backward because of the pressure gradient.

Figure $2 c$ reports the results for $\operatorname{Re}=20,000$. The patterns appear somewhat different from the previous two cases, with the presence of several low pressure regions of short extent between the slot exit and $x=1.3$. Downstream from this point irregular spots of high pressure are visible. Similar results are shown in Figure $2 d$ for $\mathrm{Re}=40,000$.

From the numerical results of Figures 1 and 2, it is clear that despite the presence of the $1 \%$ disturbance on the velocity profile at the slot exit, a region of flow free of vortices is present, as in [1], where the inlet profile was flat without turbulence. The instantaneous fields are variable and cannot be employed to identify the average length of the regions of flow. Therefore, the evolution

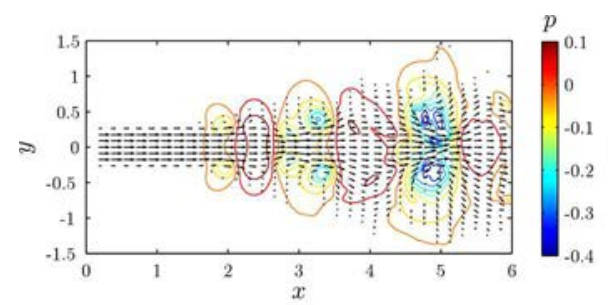

(a)

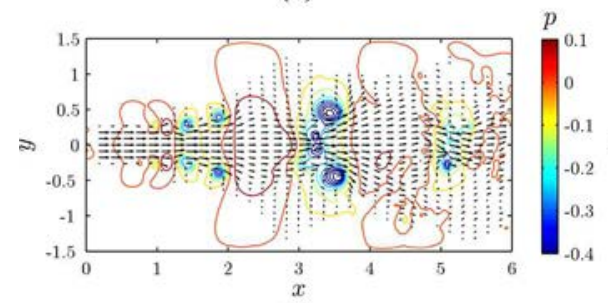

(b)

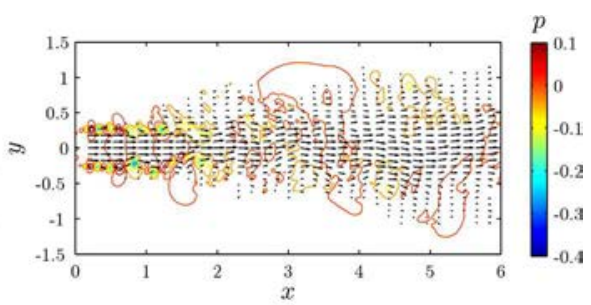

(c)

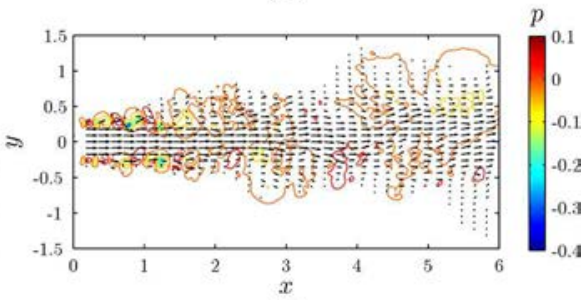

(d)

Figure 2. Instantaneous isobaric surfaces and velocity vectors: (a) $\operatorname{Re}=5,000 ;(b) \operatorname{Re}=10,000 ;(c) \operatorname{Re}=20,000 ;(d) \operatorname{Re}=40,000$. 
of some mean variables is analyzed in order to understand the average effect of the turbulence on the flow.

\subsection{Average variables}

Figure $3 a$ presents the evolution of the nondimensional average turbulent kinetic energy, $k$, for $\operatorname{Re}=5,000$. The turbulent kinetic energy is almost null between the slot exit and $x=1.5$, confirming that despite the perturbation introduced on the velocity profile at the slot exit, the turbulence develops only at a certain distance downstream, or, in other words, the region is free of turbulence and can be identified as the URF.

Turbulence originates from two points, one above and the other below the centerline, i.e., on the boundary layers between the submerged jet and the stagnant air. Moving downstream from these two points, the turbulent kinetic energy diffuses linearly up to a position where the profiles in the upper and lower parts blend together along the centerline. The region between the two point sources and the connection of the upper and lower profiles has the shape of the PCR.

Figure $3 b$ shows the turbulent kinetic energy for $\mathrm{Re}=10,000$, which is negligible close to the slot exit and starts to develop around $x=1$. Figure $3 c$ shows the $k$ contours at $\operatorname{Re}=20,000$. The beginning of the turbulent kinetic energy is located around $x=0.1$ and the diffusion in the vertical direction is smaller compared to the previous two cases. For $\mathrm{Re}=40,000$, Figure $3 d$, the $k$ profile is similar to $\operatorname{Re}=20,000$, except the source points, which are closer to the slot exit, and the diffusion in the vertical direction, which is even more limited.

Figure 4 shows the maps of the $x-y$ component of the Reynolds stress tensor, $\overline{u^{\prime} v^{\prime}}$, at the four Reynolds numbers investigated. The stress tensor is reported as positive in the upper part of the domain, and negative in the lower part.

Figure $4 a$, at $\operatorname{Re}=5,000$, shows that despite the presence of a perturbation on the velocity profile at the slot exit, the Reynolds stresses are negligible up to about $x=1.5$, which is equal to the position where turbulent kinetic energy starts developing as in Figure $2 a$. At this axial position there are two points from which the turbulent shear stress originates and spreads downstream. The contours show approximately a linear expansion in the transverse direction, up to a downstream point where the shear stresses in the upper and lower parts of the domain merge. This pattern reminds of the PCR, although this cannot be established without inspecting the velocity profile.

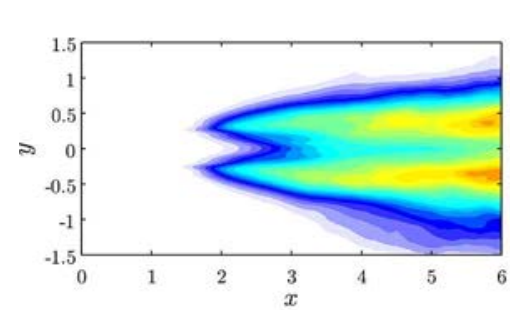

(a)

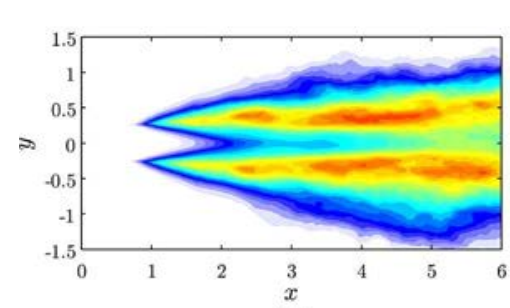

(b)
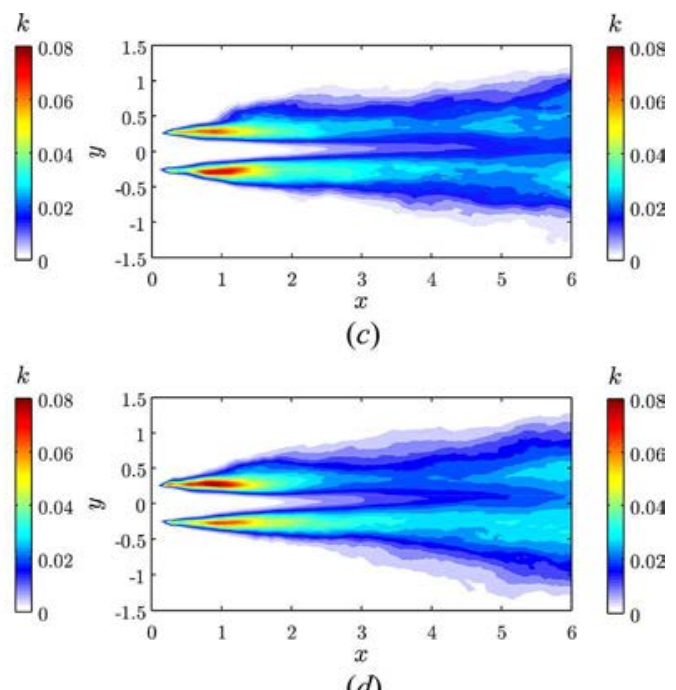

(d)

Figure 3. Turbulent kinetic energy contours: (a) $\operatorname{Re}=5,000 ;(b) \operatorname{Re}=10,000$; (c) $\operatorname{Re}=20,000$; (d) $\operatorname{Re}=40,000$. 
Figure $4 b$, at $\operatorname{Re}=10,000$, shows a pattern of $\overline{u^{\prime} v^{\prime}}$ similar to that at $\operatorname{Re}=5,000$, although the turbulent shear stress starts closer to the slot exit, in agreement with the vorticity and turbulent kinetic energy results, showing a shorter length of the URF. The linear expansion of the turbulent stresses is visible as well, which terminates earlier, and the upper and lower parts meet at a certain distance.

Figure $4 c$ shows the pattern of $\overline{u^{\prime} v^{\prime}}$ at $\operatorname{Re}=20,000$. The sources of the shear stress are now very close to the slot exit and the area of linear expansion is longer, compared to the previous two cases, terminating earlier, where the positive and the negative stresses meet. Similar considerations can be done for Figure $4 d$, where the results for $\operatorname{Re}=40,000$ are shown. The differences with $\operatorname{Re}=20,000$ are minors.

From the two average turbulent variables, i.e., $k$ and $\overline{u^{\prime} v^{\prime}}$, it is possible to distinguish between two different behaviors in the near-field region, already analyzed in [1], plus a third one in the far-field, which was not captured in the 2D simulations of [1] because of the lack of vortex stretching.

The analysis of the mean axial velocity profile, done in [1], showed the shape of the "isotach" curves in the URF and PCR. The same conclusions can be drawn from the present 3D results, which are not reported here because they are similar to [1]. The isotachs in the FDR, which could not be captured in the 2D study [1], are reported in the following sections.

\subsection{Self-similar solutions in the URF}

The analysis of the variables in the URF suggests that the dependence on the span-wise direction can be neglected. Therefore, despite the presence of a perturbation on the velocity profile at the slot exit, the flow can be considered $2 \mathrm{D}$ in the URF, and, in analogy with [1], the following relation can be obtained from the analysis of the isotach:

$$
\eta=\eta_{0}+\sqrt{\operatorname{Re}} \frac{(y-h)}{\sqrt{x}}
$$

where $h$ is the nondimensional half-slot vertical coordinate equal to 0.25 .

The analysis of the continuity and momentum equations shows that the jet flow in the URF can be described by the following ordinary differential equation:

$$
\frac{d^{3} f}{d \eta^{3}}+\frac{1}{2} f \frac{d^{2} f}{d \eta^{2}}=0
$$

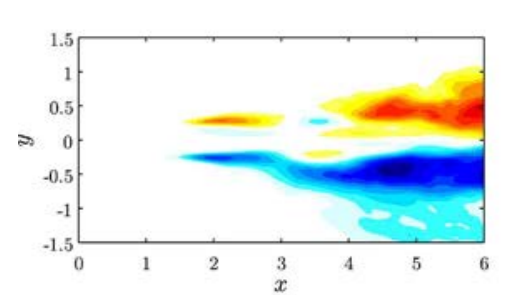

(a)

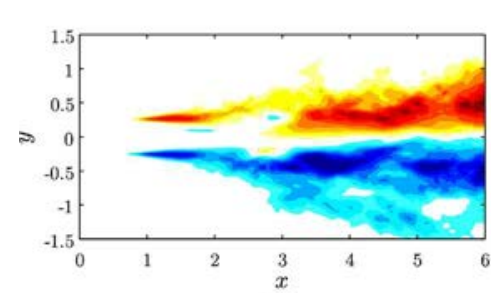

(b)
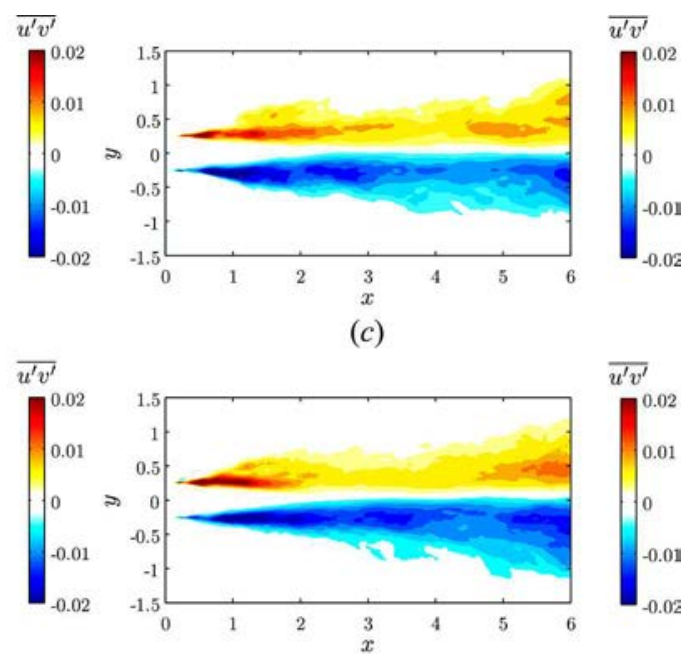

(d)

Figure 4. $\overline{u^{\prime} v^{\prime}}$ contours: (a) $\operatorname{Re}=5,000 ;(b) \operatorname{Re}=10,000$; (c) $\operatorname{Re}=20,000$; (d) $\operatorname{Re}=40,000$. 
where the axial and vertical velocities can be derived from $f(\eta)$ as

$$
\begin{gathered}
u(\eta)=\frac{d f}{d \eta} \\
v(x, \eta)=-\frac{1}{2} \frac{1}{\sqrt{\operatorname{Re}}} \frac{1}{\sqrt{x}}\left(f-\left(\eta-\eta_{0}\right) \frac{d f}{d \eta}\right)
\end{gathered}
$$

The vertical velocity on the centerline is assumed zero, which can be expressed by the following approximate condition:

$$
\lim _{\eta \rightarrow-\infty} f(\eta)=\eta-\eta_{0}
$$

The third-order ordinary differential equation can be solved by splitting in two systems of three firstorder differential equations and by imposing the continuity of $f, d f / d \eta$, and $d^{2} f / d \eta^{2}$, at $\eta=\eta_{0}$. A numerical code in Matlab is used to solve Eq. (15) through the routine "ode45", which solves the systems of nonlinear ordinary differential equations with the Runge-Kutta method of fourth order.

The results confirm the findings of [1], with $\eta_{0}=-0.5288, f\left(\eta_{0}\right)=-0.3384, d f /\left.d \eta\right|_{\eta_{0}}=0.6914$, and $d^{2} f /\left.d \eta^{2}\right|_{\eta_{0}}=-0.191$.

Figure 5 compares the numerical axial velocities, obtained from the LES, with the self-similar theoretical values, obtained from the solution of Eq. (15), at the four Reynolds numbers investigated. The numerical results, expressed in function of the self-similarity variable $\eta-\eta_{0}$, are reported at several axial coordinates in order to show that the hypothesis of self-similarity is confirmed. The velocity profile is a monotonically decreasing function, with the maximum at the center of the jet, becoming null in the stagnant air. The results show a very good agreement for the two smaller Reynolds numbers, $\operatorname{Re}=5,000$ and $\operatorname{Re}=10,000$. The agreement is fair at $\operatorname{Re}=20,000$ and less good

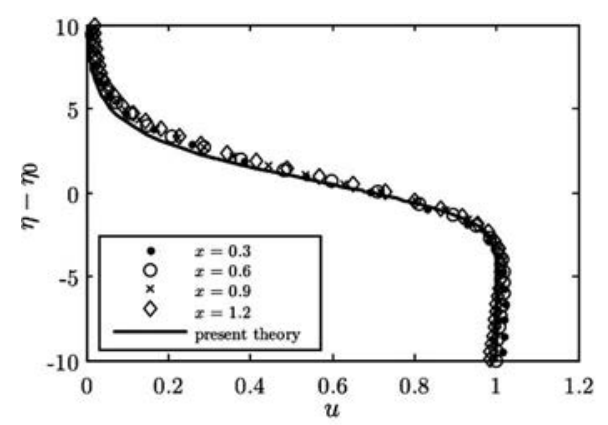

(a)

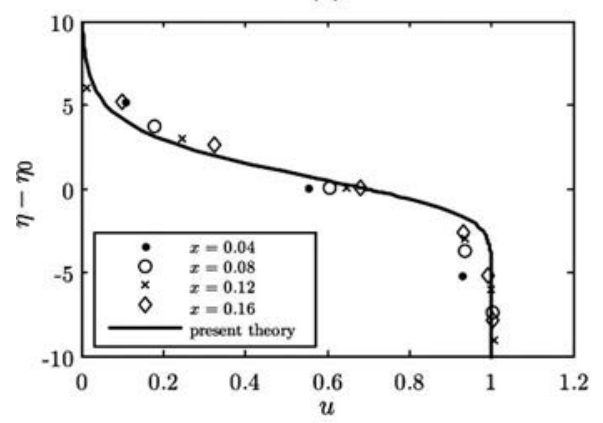

(c)

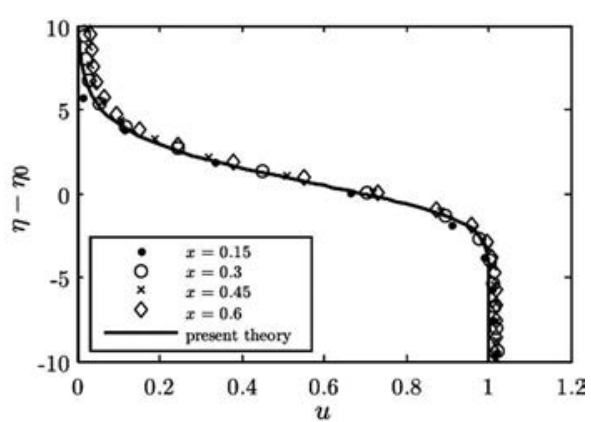

(b)

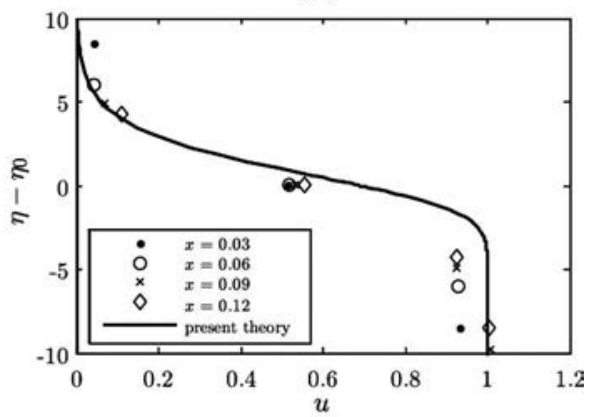

(d)

Figure 5. Axial velocity in URF: numerical (points) and present theory of self-similarity (black line): $(a) \operatorname{Re}=5,000 ;(b) \operatorname{Re}=10,000$; (c) $\operatorname{Re}=20,000 ;(d) \operatorname{Re}=40,000$. 
on the axis at the highest Reynolds number, $\mathrm{Re}=40,000$, which can be due to the perturbation imposed on the velocity profile at the slot exit, because the flow is more prone to turbulent transition.

\subsection{Self-similar solutions in the $P C R$}

The present numerical results, obtained from the LES approach, are compared with the theories of Tollmien [3] and Görtler [4], developed for the PCR. Tollmien [3] proposed the following self-similar variable:

$$
\zeta=\frac{1}{\sqrt[3]{2 c^{2}}} \frac{\left(y-h_{0}\right)}{\left(x-x_{0}\right)}
$$

which, by imposing a turbulent viscosity proportional to the square of the axial distance and the shear rate, leads to the following ordinary differential equation:

$$
\frac{d^{3} f}{d \zeta^{3}}+f=0
$$

Görtler [4] proposed the following self-similar variable:

$$
\xi=\frac{a}{e} \frac{\left(y-h_{0}\right)}{\left(x-x_{0}\right)}
$$

which, by imposing a turbulent viscosity proportional to the axial distance, leads to

$$
\frac{d^{3} f}{d \xi^{3}}+f \frac{d^{2} f}{d \xi^{2}}=0
$$

The velocities are related to the function $f$ by the following equations:

$$
\begin{gathered}
U=\frac{a}{\sqrt[3]{2 c^{2}} \frac{d f}{d \zeta}=\frac{a^{2}}{e} \frac{d f}{d \xi}} \\
V=-a\left(f(\zeta)-\zeta \frac{d f}{d \zeta}\right)=-a\left(f(\xi)-\xi \frac{d f}{d \xi}\right)
\end{gathered}
$$

The span-wise velocity in the Tollmien [3] and Görtler [4] theories is negligible. The coefficients $e$ and $c$ are nondimensional parameters directly proportional to the turbulent viscosity, found respectively in [3] and [4]. The coefficient $a$, which appears in the Eqs. (23) and (24), is assumed to be equal to 0.13 while the experimental values span between 0.09 , in [19] as well as in [2], and 0.12. From Eq. (24) it follows that the value of the constant $a$ can be deduced from the vertical velocity profile. Equations (20) and (22) are solved by providing the boundary conditions imposed in [3] and [4]. The constants can be chosen in such a way that the variables $\xi$ and $\zeta$ coincide.

The axial velocity profiles obtained from the present LES are compared with the Tollmien [3] and Görtler [4] theories in Figure 6. The numerical results are expressed in function of the similarity variable $\zeta$, and are plotted at different axial coordinates to show that the hypothesis of self-similarity is confirmed. The velocity profile in the PCR is a monotonic function of the vertical coordinate, having a maximum on the centerline and going to zero far away. Both theories give comparable results at the interface. Despite the fact that at higher Reynolds numbers the effect of the disturbances is stronger, the agreement between the present numerical results and the two theoretical values is quite good.

The comparisons, carried out in Sections 3.3 and 3.4, are important because they confirm that upstream from the fully developed zone, two self-similar regions are present: the first one follows the behavior introduced in [1] for a 2D case, while the second one follows the well-known theories of Tollmien [3] and Görtler [4]. 


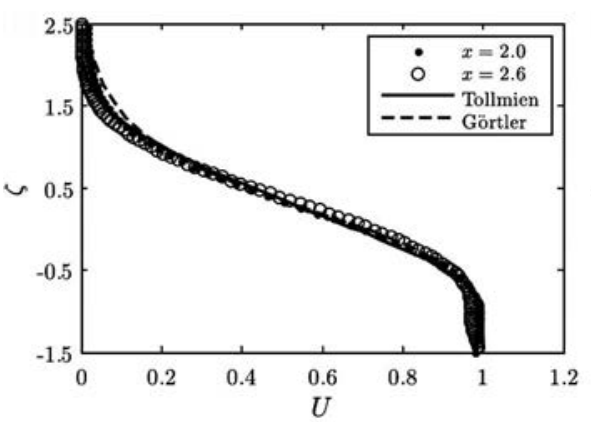

(a)

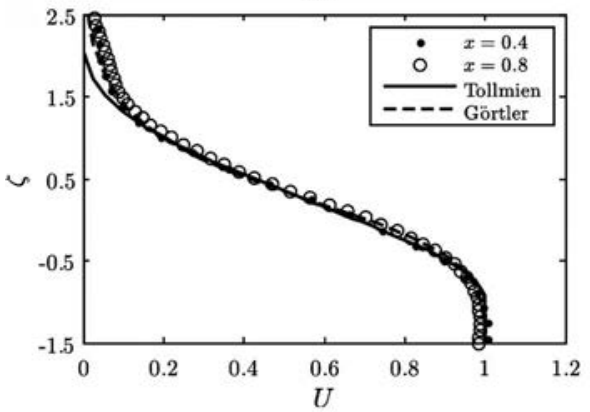

(c)

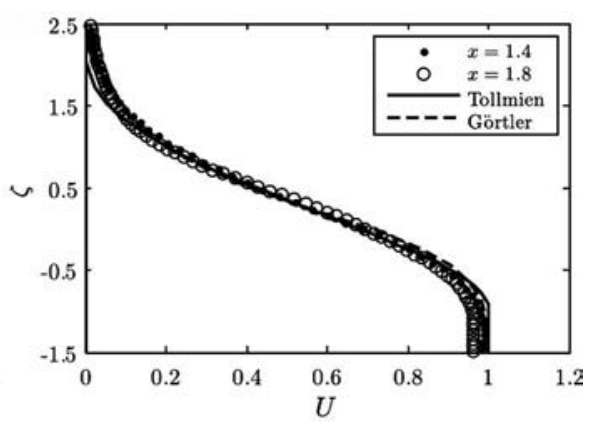

(b)

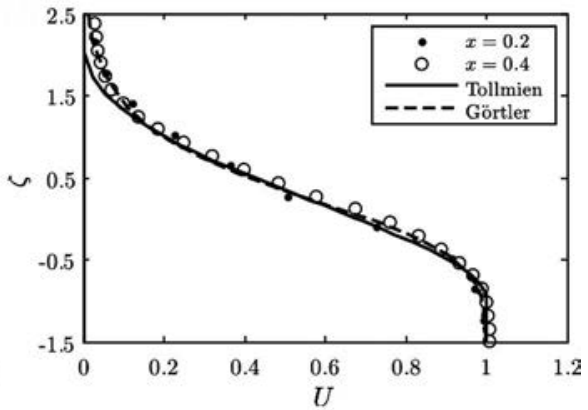

(d)

Figure 6. Axial velocity in PCR: numerical (points), Tollmien (black line), and Görtler theories (dashed line): $(a)$ Re $=5,000$; (b) $\operatorname{Re}=10,000 ;$ (c) $\operatorname{Re}=20,000 ;(d) \operatorname{Re}=40,000$.

\subsection{Self-similar solutions in the FDR}

Downstream from the PCR, the FDR begins after the point where the turbulence has penetrated into the axis. Here the mean axial velocity on the centerline, which remains constant from the slot exit, starts decreasing with the square root of the axial coordinate.

The isotach curves in the FDR, derived from the 3D numerical simulations, are reported in Figure 7. Unlike the previous regions, the slope of the isotach curves in the FDR is always positive.

The conservation of the axial and vertical momentum leads to the following equations:

$$
\begin{gathered}
U \frac{\partial U}{\partial x}+V \frac{\partial U}{\partial y}=\frac{\partial}{\partial y}\left(\nu_{T} \frac{\partial U}{\partial y}\right) \\
0=-\frac{\partial P}{\partial y}+\frac{\partial \tau_{y y}^{R}}{\partial y}
\end{gathered}
$$

The mean continuity equation is enforced by the use of the mean stream-function, $\Psi$, defined by

$$
\Psi=U_{c} \cdot \sigma \cdot\left(x-L_{s}\right) \cdot f
$$

being $f$ a function of the self-similar variable chosen, and

$$
U_{c}=\frac{n}{\sqrt{x-L_{s}}}
$$

the axial velocity at the centerline.

The theories of Tollmien [3] and Görtler [4] differ in the definition of the turbulent viscosity, which is

$$
\nu_{T}=c^{2} \cdot\left(x-L_{s}\right)^{2} \cdot\left|\frac{\partial U}{\partial y}\right|
$$




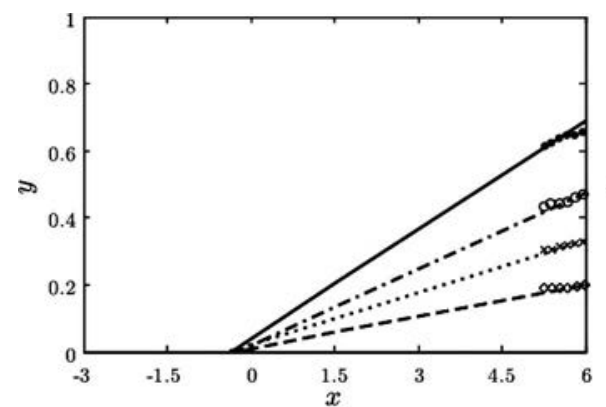

(a)

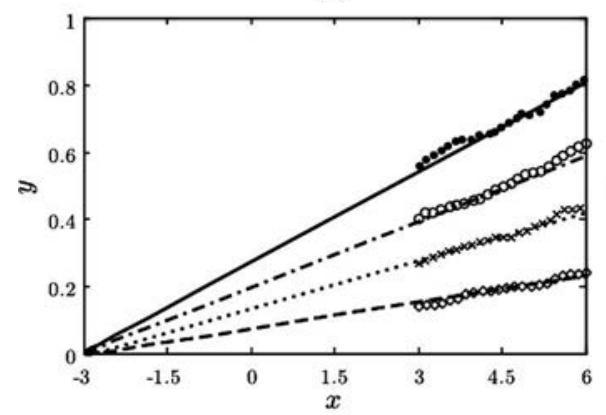

(c)

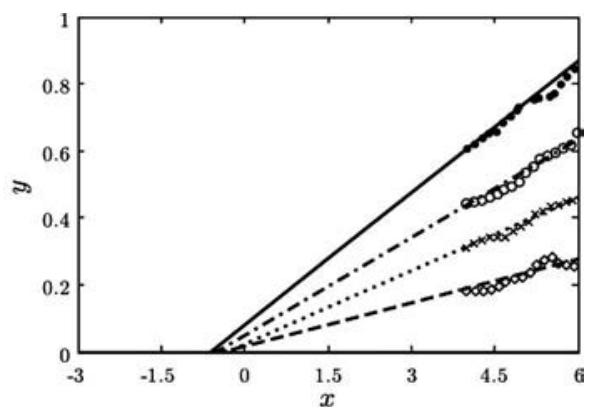

(b)

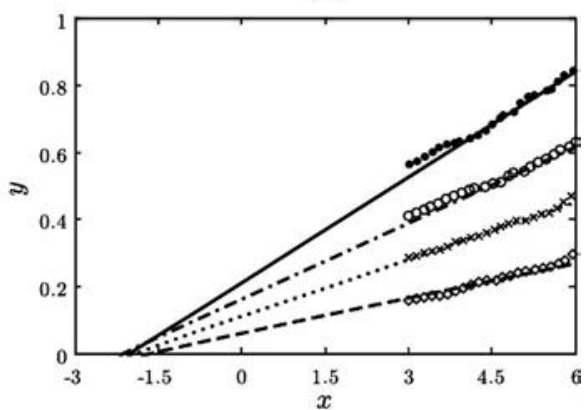

(d)

Figure 7. Numerical isotach curves in FDR. $U / U_{c}=0.3$ : numerical (black points) and regression (black line). $U / U_{c}=0.5$ : numerical (white point) and regression (dot-dashed line). $U / U_{c}=0.7$ : numerical $\left(x\right.$ point) and regression (dotted line). $U / U_{c}=0.9$ : numerical (diamond point) and regression (dashed line). (a) $\operatorname{Re}=5,000$; (b) $\operatorname{Re}=10,000$; (c) $\operatorname{Re}=20,000$; (d) $\operatorname{Re}=40,000$.

in [3], and

$$
\nu_{T}=g \cdot U_{c} \cdot\left(x-L_{s}\right)
$$

in [4].

The problem is solved in [3] by using the following self-similar variable:

$$
\zeta=\frac{1}{\sqrt[3]{2 c^{2}}} \frac{y}{\left(x-L_{s}\right)}
$$

leading to the following ordinary differential equation:

$$
\frac{d}{d \zeta}\left(\left|\frac{d^{2} f}{d \zeta^{2}}\right| \frac{d^{2} f}{d \zeta^{2}}+f \frac{d f}{d \zeta}\right)=0
$$

while the self-similar variable is defined in [4] as

$$
\xi=\frac{\sigma}{4 g} \frac{y}{\left(x-L_{s}\right)}
$$

leading to

$$
\frac{d}{d \xi}\left(\frac{d f}{d \xi}+f^{2}\right)=0
$$

The two mean velocity components are defined as

$$
U=U_{c} \frac{\sigma}{\sqrt[3]{2 c^{2}}} \frac{d f}{d \zeta}=U_{c} \frac{\sigma^{2}}{4 g} \frac{d f}{d \xi}
$$




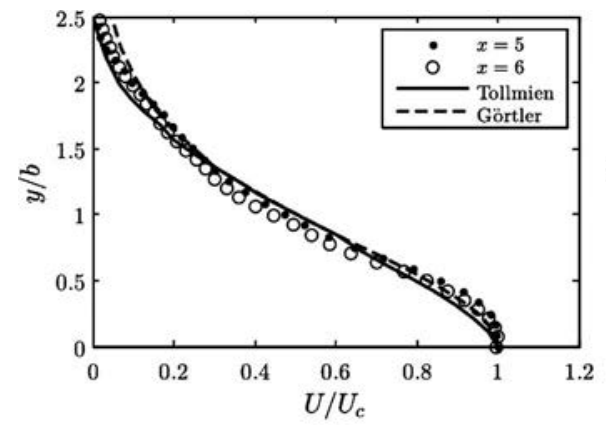

(a)

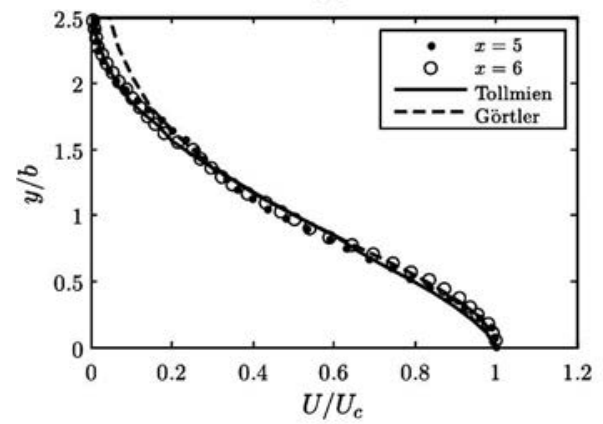

(c)

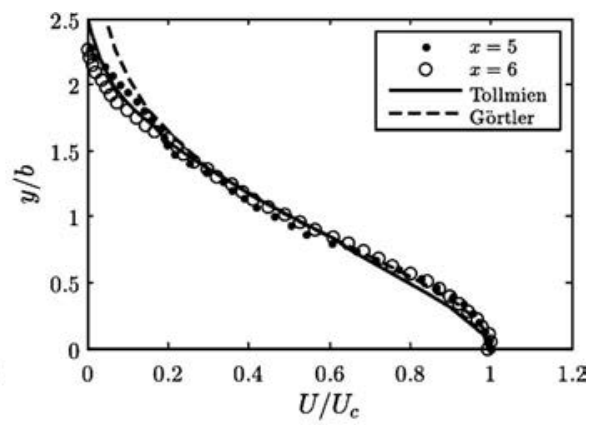

(b)

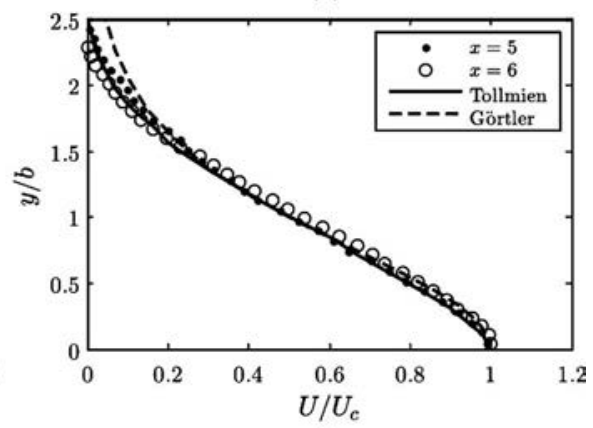

(d)

Figure 8. Axial velocity in FDR: numerical (points), Tollmien (black line), and Görtler theories (dashed line): $(a) \operatorname{Re}=5,000 ;(b)$ $\operatorname{Re}=10,000 ;(c) \operatorname{Re}=20,000 ;(d) \operatorname{Re}=40,000$.

$$
V=-U_{c} \cdot \sigma \cdot\left(\frac{1}{2} f(\zeta)-\zeta \frac{d f}{d \zeta}\right)=-U_{c} \cdot \sigma \cdot\left(\frac{1}{2} f(\xi)-\xi \frac{d f}{d \xi}\right)
$$

The constant $\sigma$ is function of the Reynolds number. Abramovich [50] has chosen values of $\sigma$ that span between 0.09 and 0.12 giving $U / U_{c}=0.5$ at $\eta=1$, while, in the present work, $\sigma$ spans between 0.06 and 0.1 . Equations (32) and (34) are solved by providing the boundary conditions imposed in [3] and [4].

The comparison between the mean axial velocity profiles, obtained from the present numerical approach and the theoretical ones [3, 4], is shown in Figure 8 at different Reynolds numbers. The velocity is scaled by its axial value and plotted versus $y / b$, where

$$
b=\sigma_{A}\left(x-L_{s}\right)
$$

and $\sigma_{A}$ is the value of the constant $\sigma$ such that $y / b(x)=1 \Rightarrow U / U_{c}=0.5$ [50].

The agreement between the present numerical results and the theoretical ones is quite good. Both the theories of [3] and [4] give comparable results at the interface, where their solutions coincide. The present numerical results are in better agreement with [3] externally, while, in the center of the jet, the agreement is better with [4], the opposite of what happens for the PCR.

\section{Conclusions}

The aim of this work is to confirm the experimental findings of [14-20] that discovered the presence of a region of flow which can be considered "undisturbed" between the exit of the slot and the beginning of the PCR. This region, or type of flow, has been called undisturbed region of flow in the average evolution, and negligible disturbance flow plus small disturbances flow in the instantaneous evolution. 
In order to confirm the experimental findings, 3D numerical simulations are carried out at several Reynolds numbers by reproducing the boundary conditions of [2]. The present 3D numerical simulations assume a top-hat velocity profile on the slot exit with a superimposed disturbance, which triggers the vortex stretching and consequently allows the FDR to appear as well. In the span-wise direction the "periodic" boundary condition has been used in order to have results independent on the slot width. The 3D numerical results are validated against the theories of Tollmien [3] and Görtler [4] for both the PCR and the FDR finding a good agreement.

The present results extend the results previously obtained in the $2 \mathrm{D}$ analysis [1]. Despite the introduction of a disturbance on the velocity profile at the slot exit, the turbulent boundary layer does not form on the slot exit. A region of flow forms between the slot exit and the PCR, where the evolution of the jet is driven by the viscous friction with the stagnant air. The length of this region of flow decreases with the increase of the Reynolds number and therefore, the theory of [2] still holds true at high Reynolds numbers, or for flows with strong perturbation added to the velocity profile at the slot exit.

Another important conclusion is that the velocity profile in the URF is self-similar, which holds true also in the 3D case with the superimposed disturbance. The evolution of the jet depends on the boundary conditions on the slot exit and a different velocity profile may produce a different flow evolution. However, since the thickness of the shear layer is proportional to the distance from the slot exit, the top-hat velocity profile is the one for which the roller-vortices appear earlier, and therefore, it is the velocity profile which produces the shortest URF. In conclusion, a different velocity profile can make the URF longer, but not shorter. Another important factor, which may affect the solution, is the turbulence intensity at the slot exit. The $1 \%$ value superimposed on the velocity profile at the slot exit in the present simulations is plausible, but in other conditions the turbulence intensity can be higher.

The present 3D numerical results, validated against the theories of [3] and [4], confirm more than a decade of experimental observations about the URF in the average flow, and the NDF and SDF in the instantaneous flow [14-20].

\section{Funding}

This project has been supported by CINECA/CASPUR with grant number std12-038.

\section{References}

[1] Andrea Boghi, Matteo Angelino, and Fabio Gori, Numerical Evidence of an Undisturbed Region of Flow in Turbulent Rectangular Submerged Free Jet, Numerical Heat Transfer, vol. 70, no. 1, pp. 14-29, 2016.

[2] M. L. Albertson, Y. B. Dai, R. A. Jensen, and H. Rouse, Diffusion of Submerged Jets, Transactions of the American Society of Civil Engineers, vol. 115, no. 1, pp. 639-64, 1950. 410.

[3] W. Tollmien, Calculation of Turbulent Expansion Processes, Zeitschrift Für Angewandte Mathematik Und Mechanik, vol. 6, no. 6, pp. 468-78, 1926.

[4] H. Gortler, Berechnung von Aufgaben Der Freien Turbulenz Auf Grund Eines Neuen Naherungsansatzes, Zeitschrift Für Angewandte Mathematik Und Mechanik, vol. 22, no. 5, pp. 244-254, 1942.

[5] E. Forthmann, Turbulent Jet Expansion, NACA-TM-789, 1936.

[6] D. R. Miller and E. W. Comings, Static Pressure Distribution in the Free Turbulent Jet, Journal of Fluid Mechanics, vol. 3, no. 1, pp. 1-16, 1957.

[7] B. G. Van der Hegge Zijnen, Measurements of the Distribution of Heat and Matter in a Plane Turbulent Jet of Air, Applied Scientific Research, vol. 7, no. 4, pp. 277-292, 1958.

[8] H. W. Liepmann and J. Laufer, Investigations of Free Turbulent Mixing, NACA Technical Note, 1947.

[9] F. O. Thomas and V. W. Goldschmidt, Structural Characteristics of a Developing Turbulent Planar Jet, Journal of Fluid Mechanics, vol. 163, pp. 227-256, 1986.

[10] L. J. S. Bradbury, The Structure of a Self-Preserving Turbulent Plane Jet, Journal of Fluid Mechanics, vol. 23, no. 01 , pp. 31-64, 1965.

[11] E. Gutmark and I. Wygnanski, The Planar Turbulent Jet, Journal of Fluid Mechanics, vol. 73, no. 3, pp. 465, 1976.

[12] A. A. Sfeir, The Velocity and Temperature Fields of Rectangular Jets, International Journal of Heat and Mass Transfer, vol. 19, no. 11, 1289-1297, 1976. 
[13] A. Krothapalli, D. Baganoff, and K. Karamcheti, On the Mixing of a Rectangular Jet, Journal of Fluid Mechanics, vol. 107, pp. 201-20, 1981.

[14] F. Gori, F. De Nigris, and E. Nino, Fluid Dynamics Measurements and Optical Visualization of the Evolution of a Submerged Slot Jet of Air, The 12th International Heat Transfer Conference, Grenoble, France, vol. 2, pp. 303-308, 2002.

[15] F. Gori and I. Petracci, Fluid Dynamics Measurements and Numerical Simulations around a Circular Cylinder Impinged by a Submerged Slot Jet of Air, American Society of Mechanical Engineers, Fluids Engineering Division 440 (Publication) FED, vol. 259, pp. 179-185, 2003.

[16] F. Gori and I. Petracci, Fluid Dynamics Measurements in a Submerged Free Slot Jet of Air, 14th International Symposium on Transport Phenomena, Bali, Indonesia, 6-10 July, pp. 491-496, 2003.

[17] F. Gori and E. Nino, Fluid Dynamics Measurements and Flow Visualizations of a Free Slot Jet of Air, American Society of Mechanical Engineers, Fluids Engineering Division (Publication), FED, vol. 259, pp. 187-192, 2003.

[18] F. Gori, E. Nino, and I. Petracci, Shadowgraph Visualizations of a Submerged Free Slot Jet of Air, International Journal of Heat and Technology, vol. 25, no. 1, pp. 157-164, 2007.

[19] F. Gori, I. Petracci, and M. Angelino, Flow Evolution of a Turbulent Submerged Two-Dimensional Rectangular Free Jet of Air. Average Particle Image Velocimetry (PIV) Visualizations and Measurements, International Journal of Heat and Fluid Flow, vol. 44, pp. 764-775, 2013.

[20] F. Gori, I. Petracci, and M. Angelino, Influence of the Reynolds Number on the Instant Flow Evolution of a Turbulent Rectangular Free Jet of Air, International Journal of Heat and Fluid Flow, vol. 50, pp. 386-401, 2014.

[21] G. B. Brown, On Vortex Motion in Gaseous Jets and the Origin of Their Sensitivity to Sound, Proceedings of the Physical Society, vol. 47, p. 703, 1935.

[22] G. S. Beavers and T. A. Wilson, Vortex Growth in Jets, Journal of Fluid Mechanics, vol. 44, pp. 97-112, 1970.

[23] A. Chambers, R. Antonia, and L. Browne, Effect of Symmetry and Asymmetry of Turbulent Structures on the Interaction Region of a Plane Jet, Experiments in Fluids, vol. 3, pp. 343-348, 1985.

[24] D. O. Rockwell and W. O. Niccolls, Natural Breakdown of Planar Jets, J. Fluids Eng., vol. 94, no. 4, pp. 720-728.

[25] S. Gogineni and C. Shih, Experimental Investigation of the Unsteady Structure of a Transitional Plane Wall Jet, Experiments in Fluids, vol. 23, no. 2, pp. 121-129, 1997.

[26] M. H. Yu, T. K. Lin, and Y. Y. Hsieh, Influence of Acoustic Forcing on the Near Field Development of a Heated Plane Jet, Experimental Thermal and Fluid Science, vol. 25, no. 1-2, pp. 13-22, 2001.

[27] F. B. Hsiao, Y. C. Lim, and J. M. Huang, On the Near-Field Flow Structure and Mode Behaviors for the RightAngle and Sharp-Edged Orifice Plane Jet, Experimental Thermal and Fluid Science, vol. 34, no. 8, pp. 1282-1289, 2010.

[28] S. Yuu, K. Ikeda, and T. Umekage, Flow-Field Prediction and Experimental Verification of Low Reynolds Number Gas-Particle Turbulent Jets, Colloids and Surfaces A: Physicochemical and Engineering Aspects, vol. 109, pp. 13-27, 1996.

[29] B. Rembold, N. A. Adams, and L. Kleiser, Direct Numerical Simulation of a Transitional Rectangular Jet, International Journal of Heat and Fluid Flow, vol. 23, no. 5, pp. 547-553, 2002.

[30] Y. Liu, P. G. Tucker, and R. M. Kerr, Linear and Nonlinear Model Large-Eddy Simulations of a Plane Jet, Computers \& Fluids, vol. 37, no. 4, May 2008, pp. 439-449.

[31] F. Gori, M. Angelino, A. Boghi, and I. Petracci, Preliminary Numerical Solutions of the Evolution of Free Jets, ASME International Mechanical Engineering Conference and Exposition, Proceedings (IMECE), vol. 7(D), pp. 463-469, 2012.

[32] F. Gori and L. Bossi, On the Cooling Effect of an Air Jet along the Surface of a Cylinder, International Communications in Heat and Mass Transfer, vol. 27, no. 5, pp. 667-676, 2000.

[33] F. Gori and L. Bossi, Optimal Slot Height in the Jet Cooling of a Circular Cylinder, Applied Thermal Engineering, vol. 23, no. 7, pp. 859-870, 2003.

[34] F. Gori and I. Petracci, Heat Transfer Measurements and Numerical Simulations in the Cooling of a Circular Cylinder by a Slot Jet of Air, American Society of Mechanical Engineers, Heat Transfer Division, (Publication) HTD, vol. 374, no. 1, pp. 3-9, 2003.

[35] B. Srinivasan and A. Dewan, LES of a Turbulent Slot Impinging Jet to Predict Fluid Flow and Heat Transfer, Numerical Heat Transfer, Part A: Applications, vol. 64, no. 10, pp. 759-776, 2013.

[36] F. Gori and P. Coppa, Circumferential Variation of Heat Transfer on Three Circular Cylinders Cooled by a Slot Jet of Air, International Journal of Heat and Technology, vol. 16, no. 2, pp. 63-69, 1998.

[37] F. Gori, I Petracci, and V. Tedesco, Cooling of Two Smooth Cylinders in Row by a Slot Jet of Air with Low Turbulence, Applied Thermal Engineering, vol. 27, no. 14-15, pp. 2415-2425, 2007.

[38] F. Gori, F. De Nigris, E. Pippione, and G. Scavarda, Cooling of Finned Cylinders by a Jet Flow of Air, American Society of Mechanical Engineers, Process Industries Division (Publication), PID, vol. 7, pp. 117-122, 2002.

[39] F. Gori, M. Borgia, and A. Doro Altan, Cooling of a Finned Cylinder by a Jet Flow of Air, American Society of Mechanical Engineers, Process Industries Division (Publication) PID, vol. 8, pp. 147-152, 2003.

[40] F. Gori, M. Borgia, A. Doro Altan, M. Mascia, and I. Petracci, Cooling of a Finned Cylinder by a Jet Flow of Air, ASME Journal of Heat Transfer, vol. 127, no. 12, pp. 1416-1421, 2005. 
[41] F. Gori, M. Mascia, and I. Petracci, Air Cooling of a Finned Cylinder with Slot Jets of Different Height, International Journal of Thermal Sciences, vol. 50, pp. 1583-1593, 2011.

[42] Ivano Petracci, Luca Manni, and Fabio Gori, Numerical Simulation of the Optimal Spacing for a Radial Finned Tube Cooled by a Rectangular Jet. I-Average Thermal Results, International Journal of Thermal Sciences, vol. 104, pp. 54-67, 2016.

[43] F. Gori and I. Petracci, Influence of Turbulence on Heat Transfer Upon a Cylinder Impinged by a Slot Jet of Air, Applied Thermal Engineering, vol. 49, pp. 106-117, 2012.

[44] Fabio Gori and Ivano Petracci, Influence of Screen Solidity Ratio on Heat Transfer Upon a Cylinder Impinged by a Rectangular Jet, International Journal of Heat and Mass Transfer, vol. 81, pp. 19-27, 2015.

[45] F. Gori and I. Petracci, On the Effect of the Slot Height in the Cooling of a Circular Cylinder with a Rectangular Jet, International Communications in Heat and Mass Transfer, vol. 48, pp. 8-14, 2013.

[46] F. Gori, I. Petracci, and M. Angelino, Experimental and Numerical Heat Transfer on a Cylinder Cooled by Two Rectangular Jets of Different Heights, ASME International Mechanical Engineering Conference and Exposition, Proceedings (IMECE), Houston, Texas, USA, vol. 7 (D), pp. 2679-2688, 2012.

[47] F. Gori, G. Pacchiarotti, and I. Petracci, Pulsating Jets Cooling Circular Cylinders, ASME International Mechanical Engineering Conference and Exposition, Proceedings (IMECE), Washington, D.C., Process Industries Division (Publication) PID, vol. 8, pp. 153-159, 2003.

[48] S. Lee, S. Lele, and P. Moin, Simulation of Spatially Evolving Compressible Turbulence and the Application of Taylors Hypothesis, Physics of Fluids A, vol. 4, pp. 1521-1530, 1992.

[49] S. Chandrasekhar, Hydrodynamic and Hydromagnetic Stability, Clarendon Press, Oxford University Press, 1961.

[50] G. N. Abramovich, The Theory of Turbulent Jets, English Translation published by M.I.T. Press, Massachusetts, 1963. 